\title{
Flexible Gas Sensor Printed on Polymer Substrate for Acetone Detection in Portable Exhaled Breath Analyzers ${ }^{+}$
}

\author{
W. Andrysiewicz ${ }^{1}$, J. Krzeminski ${ }^{2}$, K. Marszalek ${ }^{1}$, M. Sloma ${ }^{2}$ and A. Rydosz 1,* \\ 1 Department of Electronics, AGH University of Science and Technology, Al. Mickiewicza 30, Krakow, \\ Poland; Wojciech.andrysiewicz@agh.edu.pl (W.A.); Konstanty.marszalek@agh.edu.pl (K.M.) \\ 2 Warsaw University of Technology, Faculty of Mechatronics, Institute of Metrology and Biomedical \\ Engineering, Micro- and Nanotechnology Division, 8 sw. A. Boboli st., 02-525 Warsaw, Poland; \\ krzem@mchtr.pw.edu.pl (J.K.); Marcin.Sloma@mchtr.pw.edu.pl (M.S.) \\ * Correspondence: artur.rydosz@agh.edu.pl, Tel.: +48-126-172-594 \\ + Presented at the 8th GOSPEL Workshop. Gas Sensors Based on Semiconducting Metal Oxides: Basic \\ Understanding \& Application Fields, Ferrara, Italy, 20-21 June 2019.
}

Published: 19 June 2019

\section{Introduction}

Gas detectors have constantly been developed over the few last decades as a result of industrial demands: for monitoring air quality, in the automotive industry in medicine for the detection of biomarkers in several diseases. Generally, the gas sensors consists of gas sensor substrate with a gas-sensitive layer and package. The gas sensor substrates are usually realized in alumina and silicon technology and gas-sensitive layers are based on the metal oxides. Flexible gas sensor substrates are very promising in the portable exhaled breath analyzers.

\section{Material and Methods}

\subsection{Flexible Gas Sensors Substrates}

The first stage of the work was the design of the electrode, deposited on the elastic substrate. The shape of the electrode should allow receiving the signal from the sensor in a stable undisturbed manner. Therefore, a tailored design compatible with the whole sensor was developed. The designed sensor substrate consists of a comb-shaped electrode with the sensitive material deposited on the top. The change in the conductivity of the sensitive layer due to the reaction with the detected gas will change the resistance between galvanically separated electrodes. Such a construction of electrodes allows to easily apply various sensitive materials to detect the presence of gases in the atmosphere and enable easy implementation for further high throughput manufacturing of the developed sensor. Large area contact pads allow connecting the external measuring apparatus to the sensor in a reliable manner, which will increase the reliability of the solution. Figure 1 shows the designed comb-shaped electrodes. 


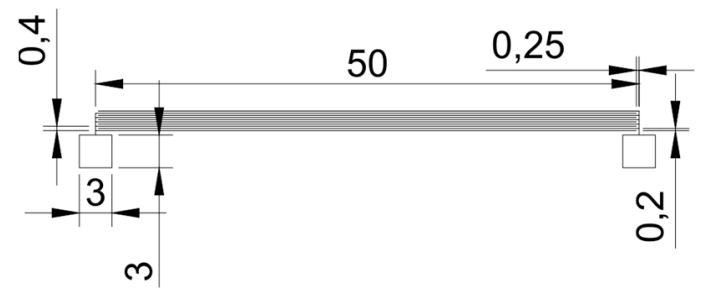

Figure 1. The designed comb-shaped electrode with dimensions in millimeters. Two contact pads 3 $\times 3 \mathrm{~mm}$ with $50 \mathrm{~mm}$ pitch, are used to connect external measuring systems. Each electrode has 5 lines with $250 \mu \mathrm{m}$ pitch.

Printed electrodes exhibit a slight non-linearity at the edge of the paths visible in Figure 2, resulting from the limitations of aerosol printing deposition. Obtained lines are homogeneous and parallel. Both the edges and corners of the contact pads were printed correctly.

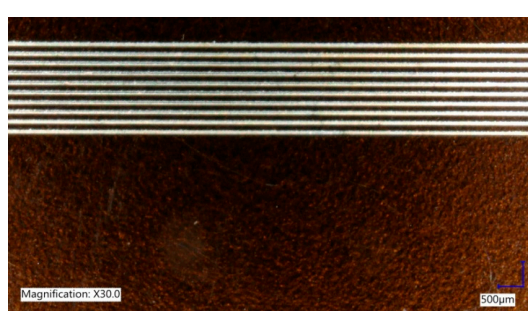

(a)

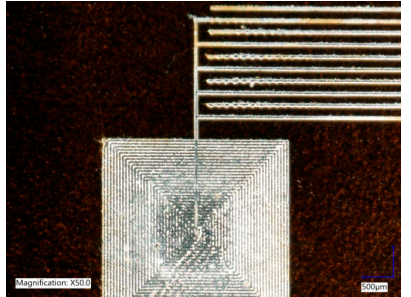

(c)

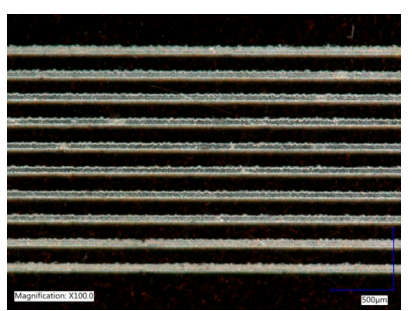

(b)

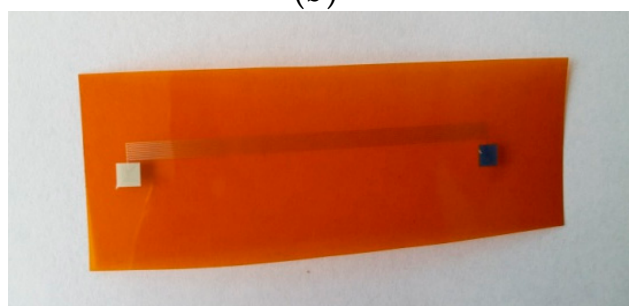

(d)

Figure 2. Micrographs of the printed sensor electrodes and contact pads; (a) general view with Magnification $\times 30.0$; (b) zoom to electrodes with Magnification $\times 100.0$; (c) zoom to contact pad with Magnification $\times 100.0$; (d) photograph of the sensor substrate.

\section{Conclusions}

Presented results demonstrate that aerosol jet printed electrodes on flexible substrates are suitable for the fabrication of gas sensors. Further perspectives are based on the patent application [1]. Flexible electronics is extremely fast developed field due to applications in screen, monitors mobiles and a few more. In our case it is applied for portable device for diabetes diagnostics. Design of multi sensors matrix on elastic substrate is a key problem for construction of such device describe in [1]. Such elastic device could be fix to a body or cloth of a patient and collects necessary data it means level of diabetic markers. Figure 3 shows a possible realization that is currently patent pending [1].

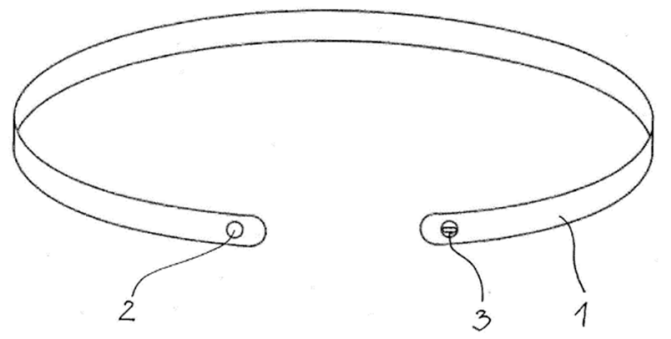

Figure 3. Drawing of the flexible exhaled breath analyzer based on the developed gas sensor substrates: (1) substrate, (2) gas-inlet port, (3) electronic port [1]. 
Funding: This research is part of the "Functional heterophase materials for structural electronics" project carried out within the First TEAM/2016-1/7 programme of the Foundation for Polish Science co-financed by the European Union under the European Regional Development Fund and partially funded by the National Science Centre, Poland 2017/26/D/ST7/00355.

Acknowledgments: This research is part of the "functional heterophase materials for structural electronics" project carried out within the First TEAM/2016-1/7 programme of the Foundation for Polish Science cofinanced by the European Union under the European Regional Development Fund and partially funded by the National Science Centre, Poland 2017/26/D/ST7/00355.

Conflicts of Interest: The authors declare no conflict of interest.

\section{Reference}

1. Rydosz, A.; Marszalek, K. Portable Device for Detection of Biomarkers in Exhaled Air and Method of Biomarker Detection in Exhaled Air. EP18190345.1, 2018.

(C) 2019 by the authors. Licensee MDPI, Basel, Switzerland. This article is an open access article distributed under the terms and conditions of the Creative Commons Attribution (CC BY) license (http://creativecommons.org/licenses/by/4.0/). 